PROCEEDINGS OF THE

AMERICAN MATHEMATICAL SOCIETY

Volume 139, Number 2, February 2011, Pages 547-558

S 0002-9939(2010)10672-X

Article electronically published on September 17, 2010

\title{
TOEPLITZ OPERATORS ON THE DIRICHLET SPACES OF PLANAR DOMAINS
}

\author{
YOUNG JOO LEE AND QUANG DIEU NGUYEN
}

(Communicated by Marius Junge)

\begin{abstract}
We study some algebraic properties of Toeplitz operators on the Dirichlet spaces of planar domains. On domains with real analytic boundary, we show that Toeplitz operators with symbol vanishing near the boundary have rank at most 1 . Moreover, we construct explicit examples of Toeplitz operators having exactly rank 1 . This is a sharp contrast to a known result on the unit disk. Also, on simply connected domains we characterize compact Toeplitz operators in terms of the boundary vanishing property of the Berezin transform of the symbol.
\end{abstract}

\section{INTRODUCTION}

Let $\Omega$ be a bounded domain in the complex plane $\mathbb{C}$ having the cone property. Recall that a domain $\Omega$ has the cone property if every point in $\Omega$ is the vertex of a cone congruent to a fixed cone in $\mathbb{C}$. In particular, every bounded domain with $C^{1}$ smooth boundary has the cone property. See Chapter 1 of 8 for more details. The Sobolev space $\mathscr{W}^{1,2}(\Omega)$ is the completion of the space of all smooth functions $f$ on $\Omega$ for which

$$
\|f\|=\left\{\left|\int_{\Omega} f d A\right|^{2}+\int_{\Omega}\left(\left|\frac{\partial f}{\partial z}\right|^{2}+\left|\frac{\partial f}{\partial \bar{z}}\right|^{2}\right) d A\right\}^{1 / 2}<\infty,
$$

where $d A$ is the usual Lebesgue measure on $\mathbb{C}$. The space $\mathscr{W}^{1,2}(\Omega)$ is a Hilbert space with the inner product

$$
\langle f, g\rangle=\int_{\Omega} f d A \int_{\Omega} \bar{g} d A+\int_{\Omega}\left(\frac{\partial f}{\partial z} \frac{\overline{\partial g}}{\partial z}+\frac{\partial f}{\partial \bar{z}} \frac{\overline{\partial g}}{\partial \bar{z}}\right) d A .
$$

See [8, Chapter 1] or [1, Chapter III] for more details and related facts. We further assume $0 \in \Omega$. Then, the Dirichlet space $\mathscr{D}(\Omega)$ is the space of all analytic functions $f \in \mathscr{W}^{1,2}(\Omega)$ such that $f(0)=0$. Then $\mathscr{D}(\Omega)$ is closed in $\mathscr{W}^{1,2}(\Omega)$; see Proposition 2 in Section 2 for details. We let $P$ be the orthogonal projection from $\mathscr{W}^{1,2}(\Omega)$ onto $\mathscr{D}(\Omega)$. Put

$$
\mathscr{M}(\Omega)=\left\{\varphi \in L^{\infty}(\Omega): \frac{\partial \varphi}{\partial z}, \frac{\partial \varphi}{\partial \bar{z}} \in L^{\infty}(\Omega)\right\}
$$

Received by the editors August 11, 2009 and, in revised form, March 10, 2010.

2010 Mathematics Subject Classification. Primary 47B35; Secondary 32A36.

Key words and phrases. Dirichlet spaces, Toeplitz operators, rank, compact operators.

(C)2010 American Mathematical Society 
where $L^{\infty}(\Omega)$ is the space of all essentially bounded measurable functions on $\Omega$ and the derivatives are taken in the distribution sense. Also, we let $\mathscr{M}_{0}(\Omega)$ be the set of all functions $\varphi \in \mathscr{M}(\Omega)$ for which

$$
\operatorname{\operatorname {esssup}}_{\operatorname{dist}(z, \partial \Omega)<\epsilon}|\varphi(z)| \rightarrow 0 \quad \text { as } \quad \epsilon \rightarrow 0,
$$

where and in what follows $\partial F$ is the topological boundary of a set $F$ and $\operatorname{dist}(z, E)$ is the Euclidean distance between a point $z$ and a set $E$.

Given $\varphi \in \mathscr{M}(\Omega)$, the Toeplitz operator $T_{u}$ with symbol $u$ is the linear operator on $\mathscr{D}(\Omega)$ defined by

$$
T_{\varphi}(f)=P(\varphi f)
$$

for functions $f \in \mathscr{D}(\Omega)$. Then, for $\varphi \in \mathscr{M}(\Omega)$, the Toeplitz operator $T_{\varphi}$ is bounded on $\mathscr{D}(\Omega)$; see Proposition 2 in Section 2.

The aim of this paper is to investigate some algebraic properties of Toeplitz operators. In the case of the unit disk $D$, G. Cao 2] proved that for a symbol $\varphi \in C^{1}(\bar{D})$, the following three conditions are all equivalent:

(a) $\varphi=0$ on $\partial D$.

(b) $T_{\varphi}=0$ on $\mathscr{D}(D)$.

(c) $T_{\varphi}$ is compact on $\mathscr{D}(D)$.

Later, this result was proved in [7] for symbols which are finite sums of finite products of harmonic functions on the unit disk. Very recently, the above characterization has been extended in 4 to more general symbols with a certain absolutely continuity condition on the unit disk.

In this paper, we consider the same characterization problem on general bounded domains. In contrast to the known result above on the unit disk, our result shows that the implication (a) $\Rightarrow$ (b) above is no longer true on certain bounded domains. For example, on a certain domain $\Omega$ with real analytic boundary, we will show that Toeplitz operators with symbol in $\mathscr{M}_{0}(\Omega)$ must have rank 0 or 1 ; see Theorem 6 , Moreover, we construct an explicit example showing that this bound can actually be attained on a family of simply connected domains with real analytic boundary; see Corollary 7

In Theorem 10, which is the main theorem of this paper, we characterize compact Toeplitz operators on simply connected domains in terms of the boundary vanishing property of the Berezin transform of the symbol. Specifically, if the domain has a Jordan curve boundary, we show the implication (c) $\Rightarrow$ (a) above still holds; see Corollary 11. Also, on the setting of the unit disk we show that our results recover the known result mentioned above.

\section{Preliminaries}

In this section, we collect some basic properties of Dirichlet spaces and Toeplitz operators which might be known somewhere. We first prove the following norm equivalence for functions in $W^{1,2}(\Omega)$ which will be useful.

Proposition 1. Let $\Omega$ be a bounded domain satisfying the cone property and $0 \in \Omega$. Then there exist constants $C_{1}, C_{2}>0$, depending only on $\Omega$, for which

$$
C_{1}\|f\|^{2} \leq \int_{\Omega}|f|^{2} d A+\int_{\Omega}\left(\left|\frac{\partial f}{\partial z}\right|^{2}+\left|\frac{\partial f}{\partial \bar{z}}\right|^{2}\right) d A \leq C_{2}\|f\|^{2}
$$

for every $f \in \mathscr{W}^{1,2}(\Omega)$. 
Proof. By an application of the Cauchy-Schwarz inequality, we only prove the second inequality. To do this, it suffices to show there exists a constant $C$, depending only on $\Omega$, such that

$$
\int_{\Omega}|f|^{2} d A \leq C|| f \|^{2}
$$

for all $f \in \mathscr{W}^{1,2}(\Omega)$. Let $f \in \mathscr{W}^{1,2}(\Omega)$ and assume $\|f\| \leq 1$ without loss of generality. Let $B \subset \Omega$ be a ball around 0 . By the generalized Poincaré inequality (see [8, Lemma 1.1.11] for example), there exist a constant $C$, depending only on $\Omega$, and a smooth function $\varphi$ with compact support in $B$ such that

$$
\int_{\Omega}|f-\alpha|^{2} d A \leq C\left(\|f\|^{2}-\left|\int_{\Omega} f d A\right|^{2}\right)
$$

where $\alpha=\int_{\Omega} f \bar{\varphi} d A$. Note that

$$
\int_{\Omega}|f|^{2} d A-2|\alpha|\left|\int_{\Omega} f d A\right| \leq \int_{\Omega}|f-\alpha|^{2} d A .
$$

Since $\|f\| \leq 1$, it follows from (2) that

$$
\begin{aligned}
\int_{\Omega}|f|^{2} d A-2|\alpha| & \leq \int_{\Omega}|f|^{2} d A-2|\alpha||| f|| \\
& \leq \int_{\Omega}|f|^{2} d A-2|\alpha|\left|\int_{D} f d A\right| \\
& \leq C .
\end{aligned}
$$

On the other hand, by the Cauchy-Schwarz inequality we have

$$
|\alpha|^{2} \leq \int_{\Omega}|\varphi|^{2} d A \int_{\Omega}|f|^{2} d A
$$

Thus

$$
\int_{\Omega}|f|^{2} d A-2\left(\int_{\Omega}|\varphi|^{2} d A\right)^{1 / 2}\left(\int_{\Omega}|f|^{2} d A\right)^{1 / 2} \leq C,
$$

which implies that $\int_{\Omega}|f|^{2} d A$ is bounded by a constant independent on $f$. The proof is complete.

Next we prove the closedness of the Dirichlet space in $\mathscr{W}^{1,2}(\Omega)$ and the boundedness of Toeplitz operators with symbol in $\mathscr{M}(\Omega)$.

Proposition 2. Let $\Omega$ be a bounded domain satisfying the cone property and $0 \in \Omega$. Then the Dirichlet space $\mathscr{D}(\Omega)$ is closed in $\mathscr{W}^{1,2}(\Omega)$. Also, for $\varphi \in \mathscr{M}(\Omega)$, the Toeplitz operator $T_{\varphi}$ is bounded on $\mathscr{D}(\Omega)$.

Proof. Let $\left\{f_{k}\right\}$ be a sequence in $\mathscr{D}(\Omega)$ converging to a function $f \in \mathscr{W}^{1,2}(\Omega)$. By Proposition 1 we have

$$
\lim _{k \rightarrow \infty} \int_{\Omega}\left|f_{k}-f\right|^{2} d A=0
$$

By a standard argument (see [3, Chapter 1, p. 8] for example), we see that $f_{k}$ converges locally uniformly to $f$. Thus $f$ is holomorphic and $f(0)=0$. Hence $f \in \mathscr{D}(\Omega)$ and then $\mathscr{D}(\Omega)$ is closed in $\mathscr{W}^{1,2}(\Omega)$.

To prove the boundedness of Toeplitz operators, let $\varphi \in \mathscr{M}(\Omega)$. By Proposition 1, one can check that there exists a constant $C$, depending only on $\varphi$ and $\Omega$, 
such that $\|\varphi f\| \leq C\|f\|$ for every $f \in \mathscr{D}(\Omega)$. Now, the result follows from the fact that

$$
\left\|T_{\varphi} f\right\|=\|P(\varphi f)\| \leq\|\varphi f\| \leq C\|f\|
$$

for every $f \in \mathscr{D}(\Omega)$. The proof is complete.

In our characterization of Toeplitz operators with symbol $\varphi \in \mathscr{M}_{0}(\Omega)$, we consider special domains that we call admissible and that contain domains with $C^{1}$ boundary. So we introduce the following piece of terminology.

Definition 3. A bounded domain $G$ in $\mathbb{C}$ is said to be admissible if there exists a sequence $\left\{G_{j}\right\}_{j \geq 1}$ of connected open subsets $G_{j}$ of $G$ satisfying the following properties:

(a) Each $\partial G_{j}$ is a piecewise smooth simple closed curve.

(b) $G_{j} \Subset G_{j+1}$ for every $j \geq 1$ and $G=\bigcup_{j=1}^{\infty} G_{j}$.

(c) $\sup _{j \geq 1}$ length $\left(\partial G_{j}\right)<\infty$.

For two admissible domains $G_{1}, G_{2}$ with $G_{1} \cap G_{2} \neq \emptyset$, it is not hard to see that $G_{1} \cup G_{2}$ and each connected component of $G_{1} \cap G_{2}$ are admissible. It is also easy to check that if $G$ is admissible, then $\partial G$ is rectifiable; i.e., $H^{1}(\partial G)<\infty$, where $H^{1}$ denotes the one dimensional Hausdorff measure. We do not know if the converse is also true.

The following shows that any bounded domain with $C^{1}$ boundary is admissible.

Proposition 4. Every bounded domain with $C^{1}$ boundary is admissible.

Proof. Let $G$ be a bounded domain with $C^{1}$ boundary. Let $\rho$ be a defining function for $G$. That is, $\rho$ is smooth on a neighborhood $U$ of $\bar{G}, G=\{z \in U: \rho(z)<0\}$ and $d \rho \neq 0$ on $\partial G$. Fix $a \in G$. By Sard's theorem, there exists a decreasing sequence $\left\{\epsilon_{j}\right\}$ of positive numbers $\epsilon_{j}$ such that

(i) $\epsilon_{j} \rightarrow 0$ as $j \rightarrow \infty$,

(ii) $\rho(a)<-\epsilon_{1}$,

(iii) $\left\{z \in G: \rho(z)=\epsilon_{j}\right\}$ is a real one dimensional smooth compact submanifold.

Denote by $G_{j}$ the connected component of $\left\{z \in G: \rho(z)<-\epsilon_{j}\right\}$ that contains $a$. Then by (iii), $\partial G_{j}$ is a smooth simple closed curve. Also, since $\left\{\epsilon_{j}\right\}$ is decreasing, clearly $G_{j} \Subset G_{j+1}$ for every $j \geq 1$. Next, we claim $G=\bigcup_{j=1}^{\infty} G_{j}$. Let $b \in G$ be any point. Since $G$ is open connected, we can take a curve $\gamma$ in $G$ connecting $a$ and $b$. By (i), we can choose $j$ large enough such that $-\epsilon_{j}>\sup _{z \in \gamma} \rho(z)$. Then $\gamma \subset G_{j}$, and hence $b \in G_{j}$, as desired. Finally, we show that $\sup _{j \geq 1}$ length $\left(\partial G_{j}\right)<\infty$. By the compactness of $\partial G$, it suffices to prove that for every $\xi \in \partial G$, there exists an open neighborhood $U_{\xi}$ of $\xi$ such that

$$
\sup _{j \geq 1} \operatorname{length}\left(U_{\xi} \cap \partial G_{j}\right)<\infty .
$$

Fix $\xi=\xi_{1}+i \xi_{2} \in \partial G$. Since $d \rho(\xi) \neq 0$, we may assume that $\frac{\partial \rho}{\partial x}(\xi) \neq 0$. By the implicit function theorem, we can find open intervals $V_{\xi}, W_{\xi}$ and a constant $t_{\xi}>0$ such that $\xi_{1} \in W_{\xi}, \xi_{2} \in V_{\xi}$ and

$$
\rho(x, y)=t,(x, y, t) \in W_{\xi} \times V_{\xi} \times\left(-t_{\xi}, t_{\xi}\right) \Leftrightarrow x=h(y, t),
$$


where $h$ is a smooth function defined on a neighborhood of $\overline{V_{\xi}} \times\left[-t_{\xi}, t_{\xi}\right]$. Set $U_{\xi}:=W_{\xi} \times V_{\xi}$ and choose $j_{0}$ such that $\epsilon_{j}<t_{\xi}$ for $j>j_{0}$. Thus for $j>j_{0}$ we have

$$
\operatorname{length}\left(U_{\xi} \cap\left\{\rho=\epsilon_{j}\right\}\right)=\int_{V_{\xi}} \psi\left(y, \epsilon_{j}\right) d y
$$

for some function $\psi$ smooth on a neighborhood of $\overline{V_{\xi}} \times\left[-t_{\xi}, t_{\xi}\right]$. It follows that length $\left(U_{\xi} \cap \partial G_{j}\right)$ is uniformly bounded for $j>j_{0}$. The proof is complete.

\section{Toeplitz operators With Symbol in $\mathscr{M}_{0}(\Omega)$}

In this section, we show that on certain admissible domain $\Omega$, Toeplitz operators with symbol in $\mathscr{M}_{0}(\Omega)$ must have rank 0 or 1 . Moreover, we give examples of concrete domains and symbols for which the ranks of the corresponding Toeplitz operators are exactly one.

Fix a nonnegative function $\phi \in C^{\infty}(\mathbb{C})$ with compact support in the unit disk $D$ such that $\int_{\mathbb{C}} \phi d A=1$. Such a function $\phi$ is called a mollifier. Given a locally integrable function $f$ on $\mathbb{C}$ which vanishes identically outside a domain $\Omega$ and $\rho>0$, we define a mollification $\mathcal{M}_{\rho}[f]$ of $f$ with respect to the mollifier $\phi$ by

$$
\mathcal{M}_{\rho}[f](z)=\frac{1}{\rho^{2}} \int_{\mathbb{C}} f(w) \phi\left(\frac{z-w}{\rho}\right) d A(w), \quad z \in \mathbb{C} .
$$

Then, it turns out that each $\mathcal{M}_{\rho}[f] \in C^{\infty}(\Omega)$. Moreover, if $G$ is a domain in $\Omega$ with $\bar{G} \subset \Omega$ and $f \in \mathscr{W}^{1,2}(\Omega)$, then

$$
\frac{\partial \mathcal{M}_{\rho}[f]}{\partial z}=\mathcal{M}_{\rho}\left[\frac{\partial f}{\partial z}\right] \rightarrow \frac{\partial f}{\partial z} \quad \text { as } \quad \rho \rightarrow 0
$$

in $L^{2}(G)$. Here and in what follows, $L^{p}(F)=L^{p}(F, d A)$ is the usual Lebesgue space on a measurable subset $F \subset \mathbb{C}$. See [1, Lemma 2.18] or [8, Section 1.1.5] for details and related facts.

The admissibility of a domain gives us a simple behavior of Toeplitz operators with symbol in $\mathscr{M}_{0}(\Omega)$ as shown in the next lemma.

Lemma 5. Let $\Omega$ be an admissible domain satisfying the cone property and $0 \in \Omega$. For $\varphi \in \mathscr{M}_{0}(\Omega)$, we have

$$
\left\langle T_{\varphi} f, g\right\rangle=\int_{\Omega} \varphi f d A \int_{\Omega} \bar{g} d A
$$

for every $f, g \in \mathscr{D}(\Omega)$ with $f, g^{\prime} \in L^{\infty}(\Omega)$.

Proof. Let $f, g \in \mathscr{D}(\Omega)$ be such that $f, g^{\prime} \in L^{\infty}(\Omega)$. Recall that

$$
\left\langle T_{\varphi} f, g\right\rangle=\int_{\Omega} \varphi f d A \int_{\Omega} \bar{g} d A+\int_{\Omega} \frac{\partial(\varphi f)}{\partial z} \overline{g^{\prime}} d A .
$$

Thus, to prove the result, it suffices to show that

$$
\int_{\Omega} \frac{\partial(\varphi f)}{\partial z} \overline{g^{\prime}} d A=0
$$

Since $\Omega$ is admissible, we can choose a sequence $\left\{G_{j}\right\}_{j \geq 1}$ of connected open subsets $G_{j}$ of $\Omega$ satisfying three conditions in Definition 3 . Note that $\frac{\partial(\varphi f)}{\partial z} \overline{g^{\prime}} \in L^{2}(\Omega)$. By 
an application of the dominated convergence theorem, we have

$$
\int_{\Omega} \frac{\partial(\varphi f)}{\partial z} \overline{g^{\prime}} d A=\lim _{j \rightarrow \infty} \int_{G_{j}} \frac{\partial(\varphi f)}{\partial z} \overline{g^{\prime}} d A
$$

Fix $j \geq 1$. For each $\rho>0$, consider the mollification $\mathcal{M}_{\rho}[\varphi f]$ of $\varphi f$ with respect to a fixed mollifier $\phi$. Note that

$$
\mathcal{M}_{\rho}[\varphi f](z)=\frac{1}{\rho^{2}} \int_{|w| \leq \rho}[\varphi f](z-w) \phi\left(\frac{w}{\rho}\right) d A(w)
$$

for points $z \in \Omega$ with $\operatorname{dist}(z, \partial \Omega)>\rho$. Then, for $\rho$ small enough, $\mathcal{M}_{\rho}[\varphi f]$ is smooth on a neighborhood of $\overline{G_{j}}$. Since $\int_{\mathbb{C}} \phi d A=1$, we have for $0<\rho<\operatorname{dist}\left(\partial G_{j}, \partial \Omega\right)$

$$
\sup _{z \in \partial G_{j}}\left|\mathcal{M}_{\rho}[\varphi f](z)\right| \leq\|\varphi\|_{L^{\infty}\left(\Omega \backslash G_{j}\right)} \sup _{z \in \Omega}|f(z)| \text {. }
$$

Furthermore, since $\frac{\partial(\varphi f)}{\partial z} \in L^{2}(\Omega)$, we note that

$$
\frac{\partial \mathcal{M}_{\rho}[\varphi f]}{\partial z} \rightarrow \frac{\partial(\varphi f)}{\partial z} \quad \text { as } \quad \rho \rightarrow 0
$$

in $L^{2}\left(G_{j}\right)$. It follows from Stoke's theorem that

$$
\begin{aligned}
\int_{G_{j}} \frac{\partial(\varphi f)}{\partial z} \overline{g^{\prime}} d A & =\lim _{\rho \rightarrow 0} \int_{G_{j}} \frac{\partial \mathcal{M}_{\rho}[\varphi f]}{\partial z} \overline{g^{\prime}} d A \\
& =\lim _{\rho \rightarrow 0} \frac{i}{2} \int_{G_{j}} \frac{\partial\left(\mathcal{M}_{\rho}[\varphi f] \overline{g^{\prime}}\right)}{\partial z} d z \wedge d \bar{z} \\
& =\lim _{\rho \rightarrow 0} \frac{i}{2} \int_{\partial G_{j}} \mathcal{M}_{\rho}[\varphi f] \overline{g^{\prime}} d \bar{z} .
\end{aligned}
$$

Therefore, by (5) we obtain

$$
\left|\int_{G_{j}} \frac{\partial(\varphi f)}{\partial z} \overline{g^{\prime}} d A\right| \leq \frac{\operatorname{length}\left(\partial G_{j}\right)}{2} \|\left.\varphi\right|_{L^{\infty}\left(\Omega \backslash G_{j}\right)} \sup _{z \in \Omega}|f(z)| \sup _{z \in \Omega}\left|g^{\prime}(z)\right| .
$$

Since $\varphi \in \mathscr{M}_{0}(\Omega)$ by the assumption and $\sup _{j \geq 1} \operatorname{length}\left(\partial G_{j}\right)<\infty$, we have

$$
\lim _{j \rightarrow \infty} \int_{G_{j}} \frac{\partial(\varphi f)}{\partial z} \overline{g^{\prime}} d A=0 .
$$

Now, combining the above with (4), we have (3), as desired. The proof is complete.

Now we are ready to prove the main result in this section asserting that Toeplitz operators with symbol in $\mathscr{M}_{0}(\Omega)$ must have rank at most 1 on certain admissible domains.

Theorem 6. Let $\Omega$ be an admissible domain satisfying the cone property and $0 \in \Omega$. Assume there exists a dense sequence $\left\{g_{k}\right\}$ in $\mathscr{D}(\Omega)$ for which $g_{k}, g_{k}^{\prime}$ are bounded for every $k \geq 1$. Then, for a symbol $\varphi \in \mathscr{M}_{0}(\Omega)$, the rank of $T_{\varphi}$ is at most 1 . Moreover, the rank of $T_{\varphi}$ is equal to 1 if and only if

$$
\int_{\Omega} \varphi g d A \int_{\Omega} h d A \neq 0
$$

for some $h, g \in \mathscr{D}(\Omega)$. 
Proof. There are two cases: Either (6) does not hold or it does hold. First suppose (6) does not hold for all $g, h \in \mathscr{D}(\Omega)$. Then, in particular,

$$
\int_{\Omega} \varphi g_{j} d A \int_{\Omega} \overline{g_{k}} d A=0
$$

for all $j, k$. Since $\varphi \in \mathscr{M}_{0}(\Omega)$, we have by Lemma 5 that

$$
\left\langle T_{\varphi} g_{j}, g_{k}\right\rangle=\int_{\Omega} \varphi g_{j} d A \int_{\Omega} \overline{g_{k}} d A=0, \quad j, k \geq 1,
$$

which implies $T_{\varphi}=0$ because $\left\{g_{k}\right\}$ is dense. Hence the rank of $T_{\varphi}$ is 0 .

Now, suppose (6) holds for some $g, h \in \mathscr{D}(\Omega)$. Then, using the density of $\left\{g_{k}\right\}$, one can choose $j_{0}, k_{0}$ such that

$$
\int_{\Omega} \varphi g_{j_{0}} d A \int_{\Omega} \overline{g_{k_{0}}} d A \neq 0
$$

By Lemma 5 we have $\left\langle T_{\varphi} g_{j_{0}}, g_{k_{0}}\right\rangle \neq 0$, and hence in particular $T_{\varphi} g_{j_{0}} \neq 0$. For each $j=1,2, \cdots$, put

$$
\alpha_{j}:=\int_{\Omega} \varphi g_{j} d A\left(\int_{\Omega} \varphi g_{j_{0}} d A\right)^{-1}
$$

and $\tau_{j}=g_{j}-\alpha_{j} g_{j_{0}}$. Note that $\tau_{j}, \tau_{j}^{\prime}$ are bounded. Since $\varphi \in \mathscr{M}_{0}(\Omega)$, we have by Lemma 5 and a choice of $\alpha_{j}$ that

$$
\left\langle T_{\varphi} \tau_{j}, g_{k}\right\rangle=\int_{\Omega} \varphi \tau_{j} d A \int_{D} \bar{g}_{k} d A=0
$$

for each $k, j$, and hence

$$
T_{\varphi} g_{j}-\alpha_{j} T_{\varphi} g_{j_{0}}=T_{\varphi} \tau_{j}=0
$$

for each $j$. Thus $T_{\varphi} g_{j}=\alpha_{j} T_{\varphi} g_{j_{0}}$ for each $j$. Since $\left\{g_{j}\right\}$ is dense and $T_{\varphi} g_{j_{0}} \neq 0$, we see that the rank of $T_{\varphi}$ must be 1 . The proof is complete.

Remark. Let $\Omega$ be a bounded simply connected domain containing 0 with real analytic boundary. Then, we can choose a conformal map $h$ from $\Omega$ onto the unit disk $D$ such that $h(0)=0$, and $h$ extends to a biholomorphic map from a neighborhood of $\bar{\Omega}$ onto a neighborhood of $\bar{D}$. Using the fact that the set $\left\{z^{n}: n=\right.$ $1,2, \cdots\}$ spans a dense subset of $\mathscr{D}(D)$, we can easily see that the set $\left\{h^{n}: n=\right.$ $1,2, \cdots\}$ spans a dense subset of $\mathscr{D}(\Omega)$. Moreover, $h^{n}$ and $\left(h^{n}\right)^{\prime}$ are all bounded for each $n$. Recall that a bounded domain with $C^{1}$ boundary has the cone property; see Chapter 1 of 8 for details. So, every bounded simply connected domain containing 0 with real analytic boundary satisfies assumptions of Theorem 6 .

Next, we construct examples of simply connected domains and symbols for which the ranks of the corresponding Toeplitz operators are exactly one.

Corollary 7. There are Toeplitz operators whose ranks are exactly one.

Proof. For $0<\varepsilon<1$, consider the domain $\Omega_{\varepsilon}$ given by

$$
\Omega_{\varepsilon}=\left\{r e^{i \theta} \in \mathbb{C}: 0 \leq r<1+\varepsilon \sin \theta, 0 \leq \theta \leq 2 \pi\right\} .
$$

Observe that each $\Omega_{\varepsilon}$ has a real analytic boundary and can be viewed as a small deformation of the unit disk $D$. By the remark above, $\Omega_{\varepsilon}$ satisfies assumptions in Theorem [6] Define a function $\varphi_{\varepsilon}$ on $\Omega_{\varepsilon}$ by

$$
\varphi_{\varepsilon}(z)=r^{4}-r^{2}(1+\varepsilon \sin \theta)^{2}
$$


for $z=r e^{i \theta} \in \Omega_{\varepsilon}$. Then $\varphi_{\varepsilon} \in \mathscr{M}_{0}\left(\Omega_{\varepsilon}\right)$. Moreover, by direct computations using polar coordinates, we can see that

$$
\int_{\Omega_{\varepsilon}} z d A=i \pi\left(\varepsilon+\frac{1}{4} \varepsilon^{3}\right)
$$

and

$$
\int_{\Omega_{\varepsilon}} z \varphi_{\varepsilon} d A=-i \pi\left(\frac{2}{5} \varepsilon+\frac{3}{2} \varepsilon^{3}+\frac{3}{4} \varepsilon^{5}+\frac{1}{32} \varepsilon^{7}\right),
$$

and hence

$$
\int_{\Omega_{\varepsilon}} z \varphi_{\varepsilon} d A \int_{\Omega_{\varepsilon}} z d A=\pi^{2} \varepsilon^{2}\left(a_{0}+a_{1} \varepsilon^{2}+a_{2} \varepsilon^{4}+a_{3} \varepsilon^{6}+a_{4} \varepsilon^{8}\right)
$$

for some positive constants $a_{0}, a_{1}, a_{2}, a_{3}$ and $a_{4}$. Thus we can choose infinitely many $\varepsilon_{0} \in(0,1)$ such that

$$
\int_{\Omega_{\varepsilon_{0}}} z \varphi_{\varepsilon_{0}} d A \int_{\Omega_{\varepsilon_{0}}} z d A \neq 0
$$

Now, using Theorem 6, we see that each $T_{\varepsilon_{0}}$ has rank 1.

\section{Compact Toeplitz operators}

In this section, we characterize compact Toeplitz operators on simply connected domains. In the proof, we will use a known result on compactness of Toeplitz operators on the Bergman space. Given a bounded domain $\Omega$ in $\mathbb{C}$, we recall that $L^{2}(\Omega)=L^{2}(\Omega, d A)$ is the usual Lebesgue space on $\Omega$. For $f \in L^{2}(\Omega)$, we let

$$
\|f\|_{2}=\left(\int_{\Omega}|f|^{2} d A\right)^{1 / 2} .
$$

The Bergman space $A^{2}(\Omega)$ is the closed subspace of $L^{2}(\Omega)$ consisting of all holomorphic functions on $\Omega$. We let $Q$ be the orthogonal projection from $L^{2}(\Omega)$ onto $A^{2}(\Omega)$. Given $\varphi \in L^{\infty}(\Omega)$, the Bergman space Toeplitz operator $T_{\varphi}^{a}$ with symbol $u$ is the linear operator on $A^{2}(\Omega)$ defined by

$$
T_{\varphi}^{a} f=Q(\varphi f)
$$

for functions $f \in A^{2}(\Omega)$. Clearly, $T_{\varphi}^{a}$ is bounded on $A^{2}(\Omega)$. Let $K(z, w)=K_{\Omega}(z, w)$ be the Bergman kernel on $\Omega$. Recall that $K(z, z)>0$ for $z \in \Omega$; see Proposition 1.4.13 of [6]. For $\varphi \in L^{\infty}(\Omega)$, the Berezin transform $\widetilde{\varphi}$ is a function on $\Omega$ defined by

$$
\widetilde{\varphi}(z)=K(z, z)^{-1} \int_{\Omega} \psi(w)|K(z, w)|^{2} d A(w), \quad z \in \Omega .
$$

The compactness of Bergman space Toeplitz operators is characterized by the boundary vanishing property of the Berezin transform as in the next lemma, where Englis [5] worked on bounded symmetric domains in $\mathbb{C}^{n}$ for operators which are finite sums of products of several Toeplitz operators. In the following, the notation $C_{0}(\Omega)$ denotes the set of all continuous functions $\varphi$ on $\Omega$ for which $\varphi(z) \rightarrow 0$ as $z \rightarrow \partial \Omega$.

Lemma 8 (Englis [5]). Let $\Omega$ be a simply connected domain and $\varphi \in L^{\infty}(\Omega)$. Then $T_{u}^{a}$ is compact on $A^{2}(\Omega)$ if and only if $\widetilde{\varphi} \in C_{0}(\Omega)$. 
In our characterization of the compactness, the following lemma will be useful. The disk version of the following was proved in Lemma 12 of [7] with a different argument.

Lemma 9. Let $\Omega$ be a bounded domain satisfying the cone property and $0 \in \Omega$. The identity operator $I: \mathscr{D}(\Omega) \rightarrow A^{2}(\Omega)$ is compact.

Proof. By Proposition 1, we see that $I$ is bounded. If a sequence of functions in $\mathscr{D}(\Omega)$ is bounded in $\mathscr{D}(\Omega)$-norm, then it is bounded in $A^{2}(\Omega)$-norm by Proposition 1 again. So, it is locally bounded and then constitutes a normal family by Montel's theorem. Hence the sequence has a subsequence converging locally uniformly in $\Omega$ to a function in $A^{2}(\Omega)$. So $I$ is compact, as desired.

Now, we are ready to characterize compact Toeplitz operators in terms of the boundary vanishing property of the Berezin transform of the symbol.

Theorem 10. Let $\Omega$ be a simply connected domain satisfying the cone property and $0 \in \Omega$. Let $\varphi \in \mathscr{M}(\Omega)$. Then $T_{\varphi}$ is compact on $\mathscr{D}(\Omega)$ if and only if $\widetilde{\varphi} \in C_{0}(\Omega)$.

Proof. Let $U$ be the differential operator given by $U f=f^{\prime}$. By Proposition 1 . we see that $U: \mathscr{D}(\Omega) \rightarrow A^{2}(\Omega)$ is bounded. Moreover, $U$ is one-to-one and onto because $\Omega$ is simply connected with 0 . Also, by simple calculations, we obtain

$$
\left\langle T_{\varphi} g, b\right\rangle=\int_{\Omega} \varphi g d A \int_{\Omega} \bar{b} d A+\left\langle U^{*} T_{\frac{\partial \varphi}{\partial z}}^{a} I g, b\right\rangle+\left\langle U^{*} T_{\varphi}^{a} U g, b\right\rangle
$$

for every $g, b \in \mathscr{D}(\Omega)$. By Proposition 1, there exists a constant $C_{1}$ depending only on $\Omega$ such that

$$
\left|\int_{\Omega} \varphi g d A \int_{\Omega} \bar{b} d A\right| \leq C_{1}\|\varphi\|_{\infty}\|g\|_{2}\|b\|
$$

for every $g, b \in \mathscr{D}(\Omega)$.

First suppose $T_{\varphi}$ is compact on $\mathscr{D}(\Omega)$. To show $\widetilde{\varphi} \in C_{0}(\Omega)$, it suffices to show the compactness of $T_{\varphi}^{a}$ on $A^{2}(\Omega)$ by Lemma 8 . Let $\left\{f_{n}\right\}$ be a sequence in $A^{2}(\Omega)$ converging weakly to 0 . We will prove that $\left\|T_{\varphi}^{a} f_{n}\right\|_{2} \rightarrow 0$ as $n \rightarrow \infty$. Put $f_{n}=U g_{n}$ for some $g_{n} \in \mathscr{D}(\Omega)$. Then clearly $g_{n}$ converges weakly to 0 in $\mathscr{D}(\Omega)$. Let $a \in A^{2}(\Omega)$ be $\|a\|_{2} \leq 1$ and choose $b \in \mathscr{D}(\Omega)$ such that $U b=a$. Then, by an application of the open mapping theorem, we have $\|b\| \leq C_{2}$ for some constant $C_{2}$ independent of $a$. It follows from (7) that

$$
\begin{aligned}
\left\langle T_{\varphi}^{a} f_{n}, a\right\rangle_{2} & =\left\langle T_{\varphi}^{a} U g_{n}, U b\right\rangle_{2} \\
& =\left\langle U^{*} T_{\varphi}^{a} U g_{n}, b\right\rangle \\
& =\left\langle T_{\varphi} g_{n}, b\right\rangle-\int_{\Omega} \varphi g_{n} d A \int_{\Omega} \bar{b} d A-\left\langle U^{*} T_{\frac{\partial \varphi}{\partial z}}^{a} I g_{n}, b\right\rangle
\end{aligned}
$$

for each $n$. By (8), we have

$$
\left|\int_{\Omega} \varphi g_{n} d A \int_{\Omega} \bar{b} d A\right| \leq C_{1}\|\varphi\|_{\infty}\left\|g_{n}\right\|_{2}\|b\| \leq C_{1} C_{2}\|\varphi\|_{\infty}\left\|g_{n}\right\|_{2}
$$

for some constant $C_{2}$ independent on $a$ and $n$. Also, note that

$$
\begin{aligned}
\left|\left\langle T_{\varphi} g_{n}, b\right\rangle\right| & \leq\left\|T _ { \varphi } g _ { n } \left|\||| b\| \leq C_{2}\left\|T_{\varphi} g_{n}\right\|\right.\right. \\
\left|\left\langle U^{*} T_{\frac{\partial \varphi}{\partial z}}^{a} I g_{n}, b\right\rangle\right| & \leq\left\|U^{*} T_{\frac{\partial \varphi}{\partial z}}^{a} I g_{n}\right\|\|b\| \leq C_{2}\left\|U^{*} T_{\frac{\partial \varphi}{\partial z}}^{a} I g_{n}\right\|
\end{aligned}
$$


for each $n$. Combining the above together, we obtain

$$
\left\|T_{\varphi}^{a} f_{n}\right\|_{2}=\sup _{\|a\|_{2} \leq 1}\left|\left\langle T_{\varphi}^{a} f_{n}, a\right\rangle_{2}\right| \leq C\left\{\left\|T_{\varphi} g_{n}\right\|+\left\|g_{n}\right\|_{2}+\left\|U^{*} T_{\frac{\partial \varphi}{\partial z}}^{a} I g_{n}\right\|\right\}
$$

for some constant $C$ independent on $n$. Since $T_{\varphi}$ is compact on $\mathscr{D}(\Omega)$ and $g_{n} \rightarrow 0$ weakly in $\mathscr{D}(\Omega)$, we have $\left\|T_{\varphi} g_{n}\right\| \rightarrow 0$ as $n \rightarrow \infty$. Also, since $I$ is compact by Lemma 9 we see that $\left\|g_{n}\right\|_{2}$ and $\left\|U^{*} T_{\frac{\partial \varphi}{\partial z}}^{a} I g_{n}\right\|$ converge to 0 as $n \rightarrow \infty$. It follows that $\left\|T_{\varphi}^{a} f_{n}\right\|_{2} \rightarrow 0$ as $n \rightarrow \infty$, as desired.

Now, suppose $\widetilde{\varphi} \in C_{0}(\Omega)$ and let $\left\{h_{n}\right\}$ be a sequence in $\mathscr{D}(\Omega)$ converging weakly to 0 . Using (7) and (8), one can see that

$$
\left\|T_{\varphi} h_{n}\right\|=\sup _{\|b\| \leq 1}\left|\left\langle T_{\varphi} h_{n}, b\right\rangle\right| \leq C\left\{\left\|h_{n}\right\|_{2}+\left\|U^{*} T_{\frac{\partial \varphi}{\partial z}}^{a} I h_{n}\right\|+\left\|U^{*} T_{\varphi}^{a} U h_{n}\right\|\right\}
$$

for each $n$. Since $T_{\varphi}^{a}$ is compact on $A^{2}(\Omega)$ by Lemma 8 , we have $\left\|U^{*} T_{\varphi}^{a} U h_{n}\right\| \rightarrow 0$ as $n \rightarrow \infty$. Also, since $I$ is compact by Lemma 9, we see that $\left\|h_{n}\right\|_{2}$ and $\left\|U^{*} T_{\frac{\partial \varphi}{\partial z}}^{a} I h_{n}\right\|$ converge to 0 as $n \rightarrow \infty$. Now, by (9), we have $\left\|T_{\varphi} h_{n}\right\| \rightarrow 0$ as $n \rightarrow \infty$, and hence $T_{\varphi}$ is compact on $\mathscr{D}(\Omega)$, as desired. The proof is complete.

Recall that the Bergman kernel $K_{D}(z, w)$ for the unit disk $D$ is given by

$$
K_{D}(z, w)=\frac{1}{\pi(1-z \bar{w})^{2}}, \quad z, w \in D
$$

and hence the Berezin transform on $D$ can be given by

$$
\widetilde{\varphi}(z)=\frac{\left(1-|z|^{2}\right)^{2}}{\pi} \int_{D} \frac{\varphi(w)}{|1-z \bar{w}|^{4}} d A(w), \quad z, w \in D
$$

for $\varphi \in L^{\infty}(D)$. For $\varphi \in C(\bar{D})$, it turns out that $\tilde{\varphi} \in C(\bar{D})$ and $\varphi-\tilde{\varphi} \in C_{0}(D)$. See Chapter 6 of 9 ] for details.

In addition, if $\partial \Omega$ is a Jordan curve and a symbol is continuous up to the boundary, our next application says that the compactness of Toeplitz operators implies the boundary vanishing property of the symbol.

Corollary 11. Let $\Omega$ be a simply connected domain satisfying the cone property, $0 \in \Omega$ and $\partial \Omega$ be a Jordan curve. Suppose $\varphi \in \mathscr{M}(\Omega) \cap C(\bar{\Omega})$. If $T_{\varphi}$ is compact on $\mathscr{D}(D)$, then $\varphi=0$ on $\partial \Omega$.

Proof. By Theorem [10, we have $\widetilde{\varphi} \in C_{0}(\Omega)$. Since $\Omega$ is simply connected with Jordan curve boundary $\partial \Omega$, we can find a conformal map $h$ from $\Omega$ onto $D$ such that $h$ continues to be a homeomorphism from $\bar{\Omega}$ onto $\bar{D}$. By the conformal invariance of the Bergman kernel (see [3, Theorem 1, p. 12] for example), we have

$$
K_{\Omega}(z, w)=\frac{1}{\pi} \frac{h^{\prime}(z) \overline{h^{\prime}(w)}}{(1-h(z) \overline{h(w)})^{2}}, \quad z, w \in \Omega,
$$

and thus

$$
\widetilde{\varphi}(z)=\frac{\left(1-|h(z)|^{2}\right)^{2}}{\pi} \int_{\Omega} \frac{\varphi(w)\left|h^{\prime}(w)\right|^{2}}{|1-h(z) \overline{h(w)}|^{4}} d A(w) .
$$

By the change of variable $\xi=h(w)$, we get by (10)

$$
\widetilde{\varphi}(z)=\frac{\left(1-|h(z)|^{2}\right)^{2}}{\pi} \int_{D} \frac{\varphi \circ h^{-1}(\xi)}{|1-h(z) \bar{\xi}|^{4}} d A(\xi)=\widetilde{\varphi \circ h^{-1}}(h(z))
$$


for $z \in \Omega$. Since $\varphi \in C(\bar{\Omega})$, we have $\varphi \circ h^{-1} \in C(\bar{D})$, and hence $\varphi \circ h^{-1}(\xi)-$ $\widetilde{\varphi \circ h^{-1}}(\xi) \rightarrow 0$ as $|\xi| \rightarrow 1$. Observe that $z \rightarrow \partial \Omega$ if and only if $|h(z)| \rightarrow 1$. Since $\widetilde{\varphi} \in C_{0}(\Omega)$, it follows that

$$
\begin{aligned}
\lim _{z \rightarrow \partial \Omega} \varphi(z) & =\lim _{z \rightarrow \partial \Omega}[\varphi(z)-\widetilde{\varphi}(z)] \\
& =\lim _{z \rightarrow \partial \Omega}\left[\varphi \circ h^{-1}(h(z))-\widetilde{\varphi \circ h^{-1}}(h(z))\right] \\
& =0,
\end{aligned}
$$

so $\varphi=0$ on $\partial \Omega$, as desired. The proof is complete.

In addition, the boundary of the domain is real analytic, and we have the following equivalence.

Corollary 12. Let $\Omega$ be a simply connected domain with real analytic boundary and $0 \in \Omega$. For $\varphi \in \mathscr{M}(\Omega) \cap C(\bar{\Omega})$, the following conditions are equivalent:

(a) $\varphi=0$ on $\partial \Omega$.

(b) The rank of $T_{\varphi}$ is 0 or 1 .

(c) $T_{\varphi}$ is compact on $\mathscr{D}(\Omega)$.

Proof. By Theorem 6 and the remark that follows, we have (a) $\Rightarrow$ (b). Clearly (b) $\Rightarrow$ (c) is trivial and (c) $\Rightarrow$ (a) follows from Corollary 11

In Corollaries 11 and 12 we do not know whether the boundary continuity condition is essential. But, on the unit disk, by a standard argument using mollification, we see that for $\varphi \in \mathscr{M}(D) \cap C(D)$,

$$
|\varphi(z)-\varphi(w)| \leq C|z-w|, \quad z, w \in D,
$$

for some constant $C$ independent on $z, w$. Thus each $\varphi \in \mathscr{M}(D)$ can be extended to a continuous function $\varphi$ (using the same notation) on $\bar{D}$. Also, by an application of the mean value property for holomorphic functions, we have $\int_{D} h d A=0$ for every $h \in \mathscr{D}(D)$. So, by Theorem 6 and Corollary 12, we obtain the following result, which extends the result of Cao on the unit disk mentioned in the introduction.

Corollary 13. Let $\varphi \in \mathscr{M}(D) \cap C(D)$. Then the following conditions are equivalent:

(a) $\varphi=0$ on $\partial D$.

(b) $T_{\varphi}=0$ on $\mathscr{D}(D)$.

(c) $T_{\varphi}$ is compact on $\mathscr{D}(D)$.

\section{ACKNOWLEDGMENTS}

This paper was written while the second-named author was a postdoc fellow at the Department of Mathematics of the Chonnam National University in the academic year 2008-2009. It is his pleasure to thank the faculty for their hospitality and the BK21 program for financial aid. He also thanks Professor Boo Rim Choe and Professor Hyungwoon Koo for stimulating discussions during his visit to Korea University in June of 2009. This work was partially supported by grant KRF2005-070-C00007. The second-named author was supported by a grant from the NAFOSTED program. 


\section{REFERENCES}

[1] R. Adams, Sobolev Spaces, Academic Press, New York, 1975. MR0450957 (56:9247)

[2] G. Cao, The compactness of Toeplitz operators on Dirichlet spaces, Acta Math. Sinica, 44(2001), 241-248. MR.1831525 (2002a:47036)

[3] P. Duren and A. Schuster, Bergman Spaces, American Mathematical Society, 2004. MR2033762 (2005c:30053)

[4] Y. Chen, Commuting Toeplitz operators on the Dirichlet space, J. Math. Anal. Appl. 357(2009), 214-224. MR2526821 (2010d:47031)

[5] M. Englis, Compact Toeplitz operators via the Berezin transform on bounded symmetric domains, Integral Equations Operator Theory 33(1999), 426-455. MR 1682815 (2000h:47050a)

[6] S. G. Krantz, Function Theory of Several Complex Variables, John Wiley and Sons, New York, 1982. MR635928 (84c:32001)

[7] Y. J. Lee, Algebraic properties of Toeplitz operators on the Dirichlet space, J. Math. Anal. Appl. 329(2007), 1316-1329. MR2296927 (2007m:47061)

[8] V. G. Mazja, Sobolev Spaces, Springer-Verlag, Berlin, 1980. MR817985 (87g:46056)

[9] K. Zhu, Operator Theory in Function Spaces, Second Edition, American Mathematical Society, 2007. MR2311536 (2008i:47064)

Department of Mathematics, Chonnam National University, Gwangue 500-757, Korea E-mail address: leeyj@chonnam.ac.kr

Department of Mathematics, Ha Noi National University of Education, 136 Xuan Thuy, Ha NoI, Vietnam

E-mail address: dieu_vn@yahoo.com 